»Hauptmoment « eintreten würde, wenn die Störung durch den Ring dasselbe nicht verdeckte.

Ausser dem in der Instruction von 1874 bezeichneten Nebenmoment »scheinbare Berühung " empfiehlt es sich noch ein zweites Nebenmoment zu beobachten: bei dem Eintritt das Verschwinden, bei dem Austritt den Beginn der permanenten Trübung der Contactstelle.

Beide Nebenmomente, die "scheintare Berihrung * sowohl wie die »letzte « bez. »erste permanente Trübung « haben für die Bestimmung der Parallaxe ganz und gar keinen unmittelbaren Werth, weil, übereinstimmend nach den Modellstudien und den Beobachtungen des wirklichen Phänomens von I 874 , die Auffassungen dieser Phasen durch verschiedene Beobachter ganz und gar nicht gleichartig sind und auch nicht durch künstliche Reductionen annähernd gleichartig gemacht werden können. Die Beobachtung jener Momente neben dem Hauptmoment hat aber das Interesse, die Amplitude des Contact-Phänomens für jeden Beobachter und damit - in Verbindung mit entsprechenden Modellbeobachtungen - eins der zur Beurtheilung der Sicherheit der Hauptbeobachtung wünschens werthen Daten zu ergeben.

b) zu $\$$ 2, Beobachtungen ad a): Ausserdem werden, mit der Glasscheibe, Beobachtungen zum Studium des Effects der Venus-Atmosphüre ausgeführt. Die Contactstelle wird hierbei von der Seite her (durch die Scheibe hindurch) mit einer Huilfslampe beleuchtet; die Beleuchtung ist so zu moderiren, dass der Atmosphären-Ring zur Zeit des »kritischen Moments « sichtbar zu werden aufhört bez. beginnt. - Zu einer. Vergleichung dieser Beobachtungen mit objectiv bestimmten Phasen (contactus verus) ist keine Einrichtung vorhanden....

d) zu $\$ 2$, Beobachtungen ad b): Die Veränderung der Ränderentfernung in $I^{\text {s }}$ beträgt diesmal zur Zeit der inneren Berührungen 0."050, zur Zeit der äusseren 0."053.

e) $z u \cdot \$ 3:$ Die Heliometer sollen zu den ContactBeobachtungen gar nicht angewandt werden, vergl. die Heliometer-Instruction für $\mathbf{I} 882$.

Jede Expedition hat für die Contact-Beobachtung dieses Mal zwei gleiche, 6 füssige Refractoren, Exp. IV, ausserdem einen $5 \mathrm{f}$. Refractor, und sind sämmtliche Refractoren mit Helioskopen, halbzölligen Ocularen und Ocularscalen versehen. Es gelten also durchweg die in $\$ 3$ für die Hauptfernröhre enthaltenen Bestimmungen.

Weitere etwa für die Ränderberührungen freie Beobachter würden die Passagen- und Universal-Instrumente benutzen können. Die Benutzung eines Collimator-Fernrohrs kann nur in dem Fall zugelassen werden, wenn die heliometrische Beobachtung des Durchgangs vollständig verloren gegangen sein und es sich dann unmittelbar vor dem Austritt aufklären sollte.

f) zu $\$ 4$ : Die Oeffnung soll nicht reducirt werden, vielmehr sind die Sonnengläser gegen zu starke Erhitzung durch möglichste Vermeidung unnöthiger Belichtung zu schuitzen. Dieselben sind übrigens in so reichlicher Zahl mitgegeben, dass die Gefahr des Zerspringens nicht allzu ängstlich vermieden $z u$ werden braucht.

Eine Ausnahme bildet nur die Beobachtung von $a_{1}$. Diese Phase ist, wenn ohne Helioskop beobachtet wird und die Luft klar ist, auf allen Stationen mit reducirter Oeffnung abzuwarten.

g) zu \& 4: Sämmtliche Refractoren haben Helioskope, zum Theil aber nicht polarisirende, sondern einfach prismatische oder die von Prof. Zenger angegebenen Doppelprismen. Die Helioskope der beiden letzteren Arten sind mit schwachen Sonnengläsern zu verbinden, die in genügender Zahl und Auswahl beigegeben sind.

k) zu $\$$ 5, ad a): Die Vorschriften betreffs der einzuhaltenden $Z$ wischenzeiten erleiden selbstverständliche Modificationen durch die dies $\mathrm{Mal} 9^{\mathrm{m}}$ kürzere Dauer des Eintritts bez. Austritts. - Vergl. auch $\$ 7$ der Heliometer-Instruction.

l) zu $\$ 6$ : Es können zu dieser Nachsuchung beliebige der Refractoren benutzt werden. Die Beschränkungen bezüglich der Zeit und der Sonnengläser bleiben aber in Kraft.

August 1882 .

A. Auwers.

\title{
Schreiben des Herrn Prof. R. Wolf, Director der Sternwarte in Zürich, an den Herausgeber.
}

Die soeben erschienene Nr. 56 meiner $\gg$ Astronomischen Mittheilungen « enthält zunächst eine von mir unternommene neue »Studie über die Sonnenfleckenperiode, mit Berücksichtigung der betreffenden Arbeiten der Herren Duponchel, Wichard, von der Gröben und Balfour Stewart«, in welcher theils über den Stand der Frage und die genannten Arbeiten kurz referirt, theils namentlich ein in der ersten Arbeit begangener fundamentaler Irrthum aufgedeckt, und sodann der Versuch gemacht wird, die hübsche Methode, welche Herr
Balfour Stewart auf die Declinations-Variationen angewandt hat, auch direct für die Darstellung der Jahres m it tel meiner ausgeglichenen Sonnenflecken-Relativzahlen zu benutzen. Für den Detail auf die Mittheilung selbst verweisend, erlaube ich mir nur in Beziehung auf den erwähnten Versuch mitzutheilen, dass er mir zwar auf den ersten Wurf kaum ein ebenso gutes Resultat ergab, als die von mir gleichzeitig nach einer etwas verschiedenen Methode aufgestellte Formel

$$
\begin{aligned}
r=42.9 & +21.2 \sin \left(1 \mathrm{I} 2.8+\frac{x}{\mathrm{IO}}\right)+2 \mathrm{I} .3 \sin \left(242^{\circ} .0+\frac{x}{\mathrm{II}}\right) \\
& +16.9 \sin \left(288^{\circ} .6+\frac{x}{\mathrm{I} 2}\right)+27.5 \sin \left(270^{\circ} .0+\frac{x}{8 \mathrm{I}}\right)
\end{aligned}
$$


welche annähernd die Relativzahl $r$ für das Jahr $n$ giebt, wenn

$$
x=(n-1820) \cdot 360^{\circ}
$$

eingesetzt wird, - dass ich aber die Hoffnung schöpfte, bei Anwendung derselben Methode auf meine Reihe der monatlichen Relativzahlen noch ein wesentlich besseres Resultat zu erzielen, und gleichzeitig gewisse fundamentale Fragen mit ziemlicher Sicherheit zu entscheiden. Die seither in dieser Richtung unternommenen neuen Rechnungen bestärken mich nun wirklich in dieser Hoffnung - jedoch sind sie noch nicht weit genug fortgeschritten, um schon jetzt näher darüber eintreten $\mathrm{zu}$ können.

In zweiter Linie bringt die neue Nummer die Reihe der von Herrn Wolfer im ersten Halbjahr 1881 erhaltenen und seither von ihm berechneten zahlreichen Sonnenflecken-
Positionen, welche natürlich keinen Auszug erlaubt; dagegen ersucht mich Herr Wolfer mitzutheilen, dass er wirklich, wie Herr Professor Spörer sofort bemerkte, bei der Berechnung »durch ein ärgerliches Versehen die unrichtigen Normalepochen angewindt habe, und dass in Folge dessen die sämmtlichen Normallängen $L$ um r $67^{\circ} .26$. vergrössert werden müssen, während dagegen alle übrigen Daten unbeeinflusst bleiben. \&

Zum Schlusse gebe ich noch eine kurze Fortsetzung des raisonnirenden Verzeichnisses der Sammlungen der Züricher Sternwarte, und es dürte vielleicht die Beschreibung eines Kreises ron Cary ein allgemeineres Interesse beanspruchen.

$$
\text { Zürich } 1882 \text { August } 15 . \quad R \text {. Wolf. }
$$

\section{New Planetary Nebulae.}

The objects mentioned below were detected by means of their spectra as seen through a direct vision prism.

\begin{tabular}{|c|c|c|}
\hline$\alpha \mathrm{I} 880.0$ & $\delta_{\mathrm{I}} 880.0$ & $\mathrm{R}$ e m a r k s. \\
\hline${ }^{1} 7^{\mathrm{h}} \quad 7^{\mathrm{m}} 20^{\mathrm{s}}$ & $-12^{\circ} 4^{6}$ & Oblong; small star just below. \\
\hline $17 \quad 5^{8} \quad 27$ & $-195^{I}$ & Small and bright. Near AOe. 17593-4. \\
\hline 191650 & $+\quad 117$ & DM. $+\mathrm{I}^{\circ} 3979$. Very bright and minute. \\
\hline
\end{tabular}
These spectra are all gaseous.

The first of these objects is a nebula found by Tempel (No. 585x in Dreyer's Supplement to G. C.). See also A. N. Vol. 102 pag. 226,227. 'T'empel's description »between two stars does not seem applicable.

The third object, from its stellar appearance in an ordinary eye piece, could be accurately observed with micrometric apparatus. The study of its possible parallax might therefore be interesting.

Harvard College Observatory Cambridge, U. S., 1882 Aug. 3.

Edward C. Pickering.

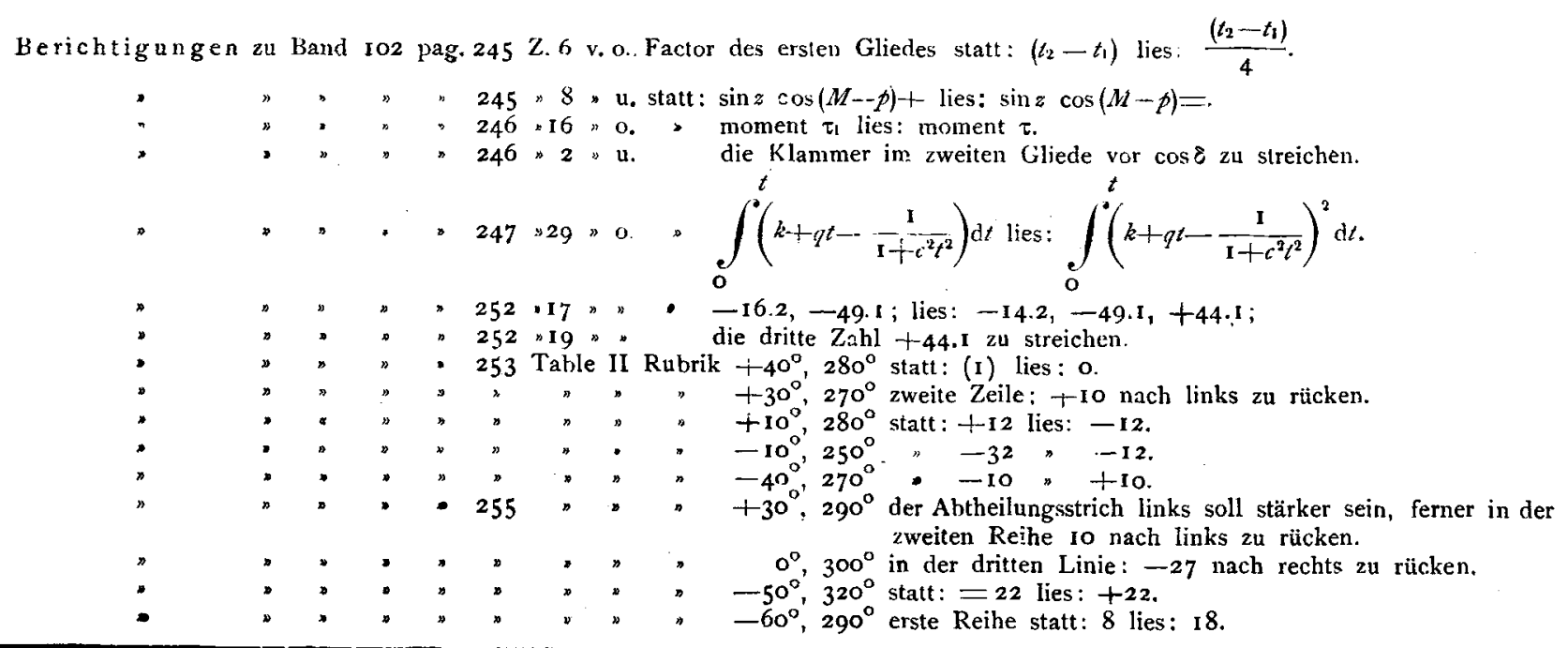

In halt zu Nr. 2454. A. Auzeers. Schreiben an den Herausgeber nebst einem Auszuge aus der lnstruction für die Beobachtung der Ränderberiuhrungen beim Venus-Durchgang. 81. $-R$. Wolf. Schreiben desselben an den Herausgeber. 93. - Edzeard C. Pickering. New Planetary Nebulae. 95. - Bericht:gungen. 95.

Geschlossen 1882 Sept. 14. Herausgeber: A. Krueger. Druck von C. F. M o hr. Frpedition: Sternwarte in Kiel. 